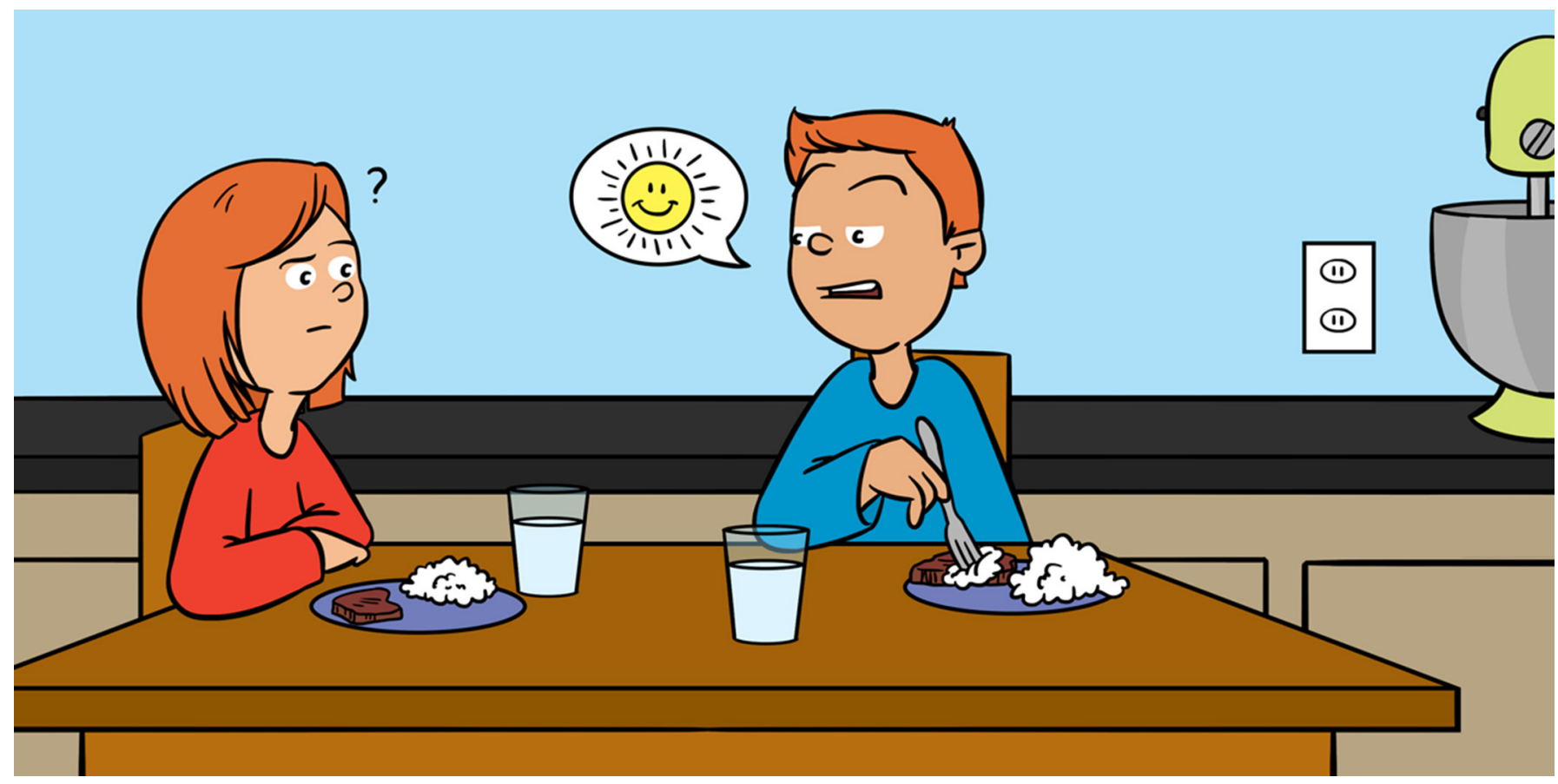

\title{
DOUBLE EMPATHY: WHY AUTISTIC PEOPLE ARE OFTEN MISUNDERSTOOD
}

\section{Catherine J. Crompton ${ }^{1 *}$, Kilee DeBrabander ${ }^{2}$, Brett Heasman ${ }^{3}$, Damian Milton ${ }^{4}$ and Noah J. Sasson ${ }^{2}$}

${ }^{1}$ Patrick Wild Centre, Division of Psychiatry, University of Edinburgh, Edinburgh, United Kingdom

${ }^{2}$ School of Behavioral and Brain Sciences, The University of Texas at Dallas, Dallas, TX, United States

${ }^{3}$ School of Psychology, York St John University, York, United Kingdom

${ }^{4}$ Tizard Centre, University of Kent, Canterbury, United Kingdom

\section{YOUNG REVIEWERS:}

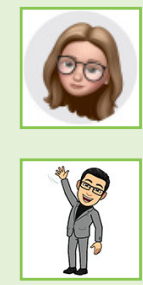

AMELIA

AGE: 15

ANAND

AGE: 13
Autism affects how someone makes sense of the world around them. About 1-2\% of people are autistic. You might have an autistic classmate or family member, or maybe you are autistic. Autistic people might communicate differently than people who are not autistic. This means that it can be difficult for other people to understand what autistic people are trying to say or what they mean. We tend to think that people who are not autistic might be more successful at understanding other people, but in fact, autistic people may be better understood by other autistic people. We will examine and explain some research that has explored how autistic and non-autistic people communicate with each other and explore how this research fits with a theory called the double empathy problem. Understanding what makes interaction comfortable and easy for different people can help us all understand each other better. 


\section{AUTISTIC}

Being autistic is considered clinically to be a medical condition but is also a source of social identity. Being autistic affects how someone makes sense of the world. Some autistic people can find it hard to communicate with other people, and might have difficulty making friends and fitting in. Autistic people might find changes difficult and might experience sounds, smells, and other senses differently. Some autistic people might move in a certain way (like twirling) or do the same thing repeatedly (like opening and closing doors). People are born autistic and remain so their whole lives. Some autistic people need only a little support, while others need a lot of help with learning and everyday activities

\section{THEORY}

An explanation for how things work and why they happen. Scientists develop theories based on observations of the world and then test these theories using research studies.

DOUBLE EMPATHY PROBLEM

A theory that helps to describe what happens when autistic and non-autistic people struggle to understand each other.

\section{EMPATHY}

The ability to understand and share the feelings of another person.

\section{WHAT IS THE DOUBLE EMPATHY PROBLEM?}

Can you tell when somebody is bored or frustrated or upset with you, even when they do not say so? People often communicate information about themselves without even saying a word. The expressions on their faces or the ways they are acting can be big clues to what they might be feeling or thinking. Being autistic affects how people make sense of the world around them, and some autistic people can find it hard to communicate. For a long time, research has shown that autistic people can have trouble figuring out what non-autistic people are thinking and feeling, and this can make it difficult for them to make friends or to fit in. But recently, studies have shown that the problem goes both ways: people who are not autistic also have trouble figuring out what autistic people are thinking and feeling! It is not just autistic people who struggle.

A theory that helps to describe what happens when autistic and non-autistic people struggle to understand each other is called the double empathy problem [1] (Figure 1). Empathy is defined as the ability to understand or be aware of the feelings, thoughts, and experiences of others. According to the double empathy problem, empathy is a two-way process that depends a lot on our ways of doing things and our expectations from previous social experiences, which can be very different for autistic and non-autistic people. These differences can lead to a breakdown in communication that can be distressing for both autistic and non-autistic people. It might sometimes be difficult for non-autistic parents to understand what their autistic child is feeling, or autistic people might feel frustrated when they cannot effectively communicate their thoughts and feelings to others. In this way, communication barriers between autistic and non-autistic people can make it more difficult for them to connect, share experiences, and empathize with one another.

Let us look at the example of "reading between the lines." This is when you understand something that someone means, even when they have not said it with words. For example, your friend might say that his day has been okay, but sigh and seem a bit grumpy or sad. Reading between the lines, you might guess that your friend's day has not been okay at all. Autistic people might struggle to read between the lines of what non-autistic people are saying, because this way of communicating does not come easily to autistic people. On the other hand, non-autistic people might make incorrect assumptions about autistic people because they are reading between the lines too much.

Autistic people can find it exhausting and confusing to understand non-autistic ways of communicating. Likewise, non-autistic people might feel uncomfortable when they are around autistic people because their usual ways of communicating do not work as well. This 
Figure 1

\section{Autistic}

and non-autistic people can find it difficult to understand each other. The fact that both people in the interaction have trouble with understanding is why the theory is called the double empathy problem.

\section{FIRST IMPRESSION}

The mental judgement made when someone encounters another person for the first time. First impressions help a person decide whether they want to be friends with or see that other person again.

\section{THE DOUBLE EMPATHY PROBLEM}
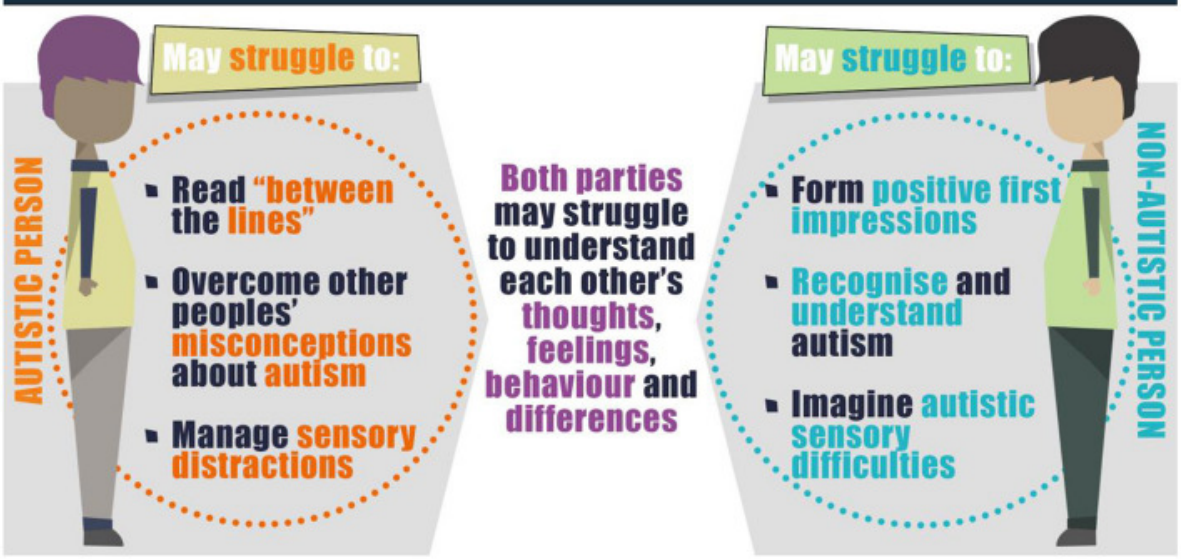

Figure 1

mismatch between social expectations and experiences can make communication between autistic and non-autistic people difficult. That is why building understanding and empathy is described as a "double problem," because both autistic and non-autistic people struggle to understand each other.

\section{WHAT HAS RESEARCH TOLD US SO FAR?}

One way that scientists understand double empathy is to see if people who are not autistic judge autistic and non-autistic people differently. Unfortunately, when people who are not autistic find it hard to understand autistic people, they tend to like them less [2]. In fact, it takes just a few seconds for people who are not autistic to form negative first impressions about autistic people [3]. Non-autistic people quickly become less interested in interacting with autistic people than with other non-autistic people, which means that autistic people may have fewer opportunities to meet people and make friends. Why does this happen? It is not because autistic people talk about things that are less interesting. When non-autistic people read the words of what autistic people are saying, they do not judge them any differently than they judge non-autistic people [3]. So, it really seems that it is how autistic people appear and sound, and not what they talk about, that leads non-autistic people to judge and avoid autistic people. Sadly, this means that autistic people might have fewer opportunities to make friends or get jobs because of how non-autistic people judge them, which is not fair.

Another way to explore double empathy is to see if autistic people connect with other autistic people more easily than they do with people who are not autistic. This is exactly what some new studies are showing. Autistic people want to talk to other autistic people, 
sit next to them, or live near them even more than they want to do these things with non-autistic people [4]. In one study, two unfamiliar adults got to know each other by talking for $5 \mathrm{~min}$ [5]. Sometimes the two adults were both non-autistic, sometimes both autistic, and sometimes one of each. You might expect, if autistic people are poor at social interaction, that the conversations between two autistic people would go especially badly. But that is not what the study found. The quality of interactions between two autistic people was just as strong as between two people who were not autistic. Autistic people even shared more information about themselves with other autistic people, suggesting they felt more comfortable with them. This shows that autistic people are like everyone else: they find it easier to connect with, and maybe even form friendships with, people who think and communicate like they do.

Why might autistic people find it easier to understand other autistic people? Research indicates that autistic people are less likely to rely on typical social expectations for interacting, or be upset if such expectations are not followed [6,7]. This means that autistic people give each other more freedom to express themselves in unique ways. We can see evidence of this by looking at how well autistic and non-autistic people share information with each other. One recent study was based on the game "Telephone," in which one person whispers a message to another person, who then whispers it to the next person, and so on. The last person then says the message out loud to see how different it is from what the first person said. Researchers compared how accurately groups of autistic people, groups of non-autistic people, and groups with a mix of autistic and non-autistic people shared a story in a game of Telephone [8]. They found that autistic groups share information just as accurately as non-autistic groups. Mixed groups of autistic and non-autistic people were much less accurate. This shows that autistic people are just as able to share information as non-autistic people if they are with other autistic people. This supports the theory of the double empathy problem: that there is a two-way difficulty when autistic and non-autistic people interact.

\section{WHAT DO WE STILL NEED TO LEARN ABOUT THE DOUBLE EMPATHY PROBLEM?}

So far, studies of the double empathy problem have focused mainly on teenagers and adults, and it will be important to see if the results differ for younger children. For example, if it turns out that non-autistic children are more positive about autistic people than non-autistic adults are, this would tell us that negative attitudes about autistic people are not destined to happen but are learned over time. Also, because autistic children are more likely now than in the past to be included in classes and activities with non-autistic children, this may provide more opportunities for autistic and non-autistic 
INTELLECTUAL DISABILITY

A disability characterized by difficulty with learning. A person with an intellectual disability might take longer to learn and may need support when developing new skills, understanding information, and interacting with others. children to interact and learn about one another. These increased opportunities for interaction might help autistic and non-autistic people to understand each other and decrease the double empathy problem over time.

Additionally, studies so far have only included autistic people who are highly verbal and do not have an intellectual disability. According to the theory, the double empathy problem would be even greater between non-autistic people and autistic people who have an intellectual disability, but further research is needed to see if this is the case. Another avenue of research is to explore the effect of familiarity of a relationship. For example, how does the double empathy problem differ when communicating with a stranger vs. a family member? Family members share backgrounds, experiences, and environments, which suggests that the double empathy problem may be reduced within familiar relationships. However, research has shown that familiarity can sometimes create additional barriers. For example, thinking we know someone well might prevent us from listening and understanding what is really being communicated [9].

Finally, although new research suggests that autistic people may communicate more effectively and more comfortably with other autistic people, we do not yet know exactly how or why this occurs. The double empathy theory would suggest that having similar ways of understanding the world helps people understand each other and connect. Understanding whether there are specific ways of communicating that underlie this connection could help us identify ways to bridge the gap in communication between autistic and non-autistic people.

\section{WHY IS THIS RESEARCH IMPORTANT?}

Social interactions are a gateway to many things in life-from buying a bus ticket to interviewing for a job. Because most people are not autistic, most social interactions fit the non-autistic communication style but might not work as well for autistic people. Autistic people must navigate many social interactions that are difficult to understand.

By finding out more about how the double empathy problem plays out in real life, we can help non-autistic and autistic people to understand each other better and help them to "meet in the middle." Improving our understanding of the ways that autistic and non-autistic people interact might help autistic people to find it easier to spend time with non-autistic friends and family as well as non-autistic teachers, doctors, and employers. It may help people who are not autistic not to leap to conclusions based on assumptions about autistic people and to be less judgmental of them. This research may also provide people who are not autistic with more creative and accessible ways of 
communicating with others. Overall, for both people who are autistic and those who are not, understanding how each other communicates can help us build understanding and make the world more inclusive and accepting of everyone-and that is important!

\section{ACKNOWLEDGMENTS}

The authors would like to give special thanks to Joe Cebula (aged 12), Minny Fletcher-Watson (aged 10), Sophie Morrison (aged 10), and Abe Sasson (aged 9) for their help in making our article more accessible to a young audience.

\section{REFERENCES}

1. Milton, D. E. M., Heasman, B., and Sheppard, E. 2020. "Double empathy," in Encyclopedia of Autism Spectrum Disorders, ed F. R. Volkmar (New York, NY: Springer). p. 1-9. doi: 10.1007/978-1-4614-6435-8_102273-2

2. Alkhaldi, R. S., Sheppard, E., and Mitchell, P. 2019. Is there a link between autistic people being perceived unfavorably and having a mind that is difficult to read? J. Autism Dev. Disord. 49:3973-82. doi: 10.1007/s10803-019-04101-1

3. Sasson, N. J., Faso, D. J., Nugent, J., Lovell, S., Kennedy, D. P., and Grossman, R. B. 2017. Neurotypical peers are less willing to interact with those with autism based on thin slice judgments. Sci. Rep. 7:40700. doi: 10.1038/srep40700

4. DeBrabander, K. M., Morrison, K. E., Jones, D. R., Faso, D. J., Chmielewski, M., and Sasson, N. J. 2019. Do first impressions of autistic adults differ between autistic and nonautistic observers? Autism Adulthood 1:250-7. doi: 10.1089/ aut.2019.0018

5. Morrison, K. E., DeBrabander, K. M., Jones, D. R., Faso, D. J., Ackerman, R. A., and Sasson, N. J. 2019. Outcomes of real-world social interaction for autistic adults paired with autistic compared to typically developing partners. Autism 24:1067-80. doi: 10.1177/1362361319892701

6. Heasman, B., and Gillespie, A. 2019. Neurodivergent intersubjectivity: distinctive features of how autistic people create shared understanding. Autism 23:910-21. doi: $10.1177 / 1362361318785172$

7. Crompton, C. J., Hallett, S., Ropar, D., Flynn, E., and Fletcher-Watson, S. 2020. 'I never realised everybody felt as happy as I do when I am around autistic people': a thematic analysis of autistic adults' relationships with autistic and neurotypical friends and family. Autism 24:1438-48. doi: 10.1177/1362361320908976

8. Crompton, C. J., Ropar, D., Evans-Williams, C. V., Flynn, E. G., and Fletcher-Watson, S. (2020). Autistic peer-to-peer information transfer is highly effective. Autism 24:1704-12. doi: 10.1177/1362361320919286

9. Heasman, B., and Gillespie, A. 2018. Perspective-taking is two sided: Misunderstandings between people with Asperger's syndrome and their family members. Autism 22:740-50. doi: 10.1177/1362361317708287

SUBMITTED: 23 April 2020; ACCEPTED: 09 April 2021;

PUBLISHED ONLINE: 11 May 2021. 
EDITED BY: Eilidh Cage, University of Stirling, United Kingdom

CITATION: Crompton CJ, DeBrabander K, Heasman B, Milton D and Sasson NJ (2021) Double Empathy: Why Autistic People Are Often Misunderstood. Front. Young Minds 9:554875. doi: 10.3389/frym.2021.554875

CONFLICT OF INTEREST: The authors declare that the research was conducted in the absence of any commercial or financial relationships that could be construed as a potential conflict of interest.

COPYRIGHT @ 2021 Crompton, DeBrabander, Heasman, Milton and Sasson. This is an open-access article distributed under the terms of the Creative Commons Attribution License (CC BY). The use, distribution or reproduction in other forums is permitted, provided the original author(s) and the copyright owner(s) are credited and that the original publication in this journal is cited, in accordance with accepted academic practice. No use, distribution or reproduction is permitted which does not comply with these terms.

\section{YOUNG REVIEWERS}

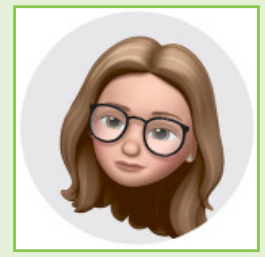

\section{AMELIA, AGE: 15}

I am studying for my GCSEs at the local high school in Northumberland, England and I am enjoying all my subjects, both arts and humanities as well as the sciences. I am particularly interested in language and how people think, what makes people different, how different people react in different situations and what are people's motivations for their actions. I also love reading and language-how it changes and evolves. I am currently hoping to study psychology and/or linguistics at university.

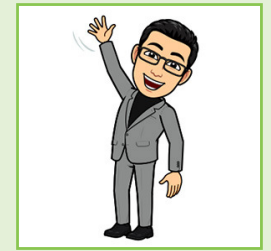

\section{ANAND, AGE: 13}

An avid learner of science and history, Anand enjoys biology and health science. His specific areas of interest are neuroscience and neurosurgery. Outside of academics, Anand's hobbies includes participating in spelling bees and learning more about roller coasters. He is a black belt in Tang So Do karate.

\section{AUTHORS}

\section{CATHERINE J. CROMPTON}

Catherine Crompton is a researcher in Psychiatry at the University of Edinburgh. She is interested in understanding the diverse ways that people communicate, and particularly how autistic people communicate. *catherine.crompton@ed.ac.uk

\section{KILEE DEBRABANDER}

Kilee DeBrabander is a researcher at The University of Texas at Dallas who studies adults with autism and how they are judged based on various characteristics of their personalities and abilities. She also looks at how these judgements may differ 

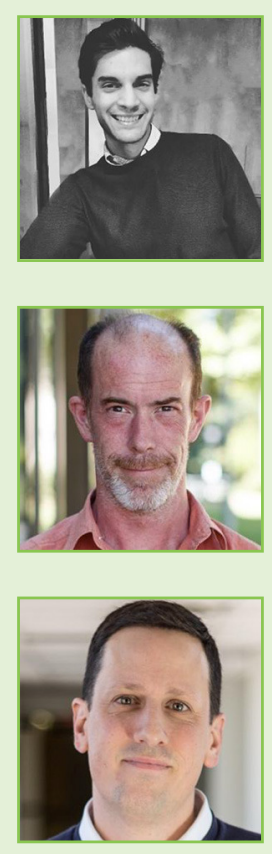

depending on if they tell other people about their autism diagnosis, if they are in a social situation or a work/school setting, or if the person making the judgements knows more about what autism is.

\section{BRETT HEASMAN}

Brett Heasman is a Senior Lecturer in Psychology at York St John University. He is interested in understanding communication, interaction, and how to remove the barriers that face people who have hidden differences.

\section{DAMIAN MILTON}

Damian Milton is a lecturer at the Tizard Centre, University of Kent and is an expert in autism research. He is interested in exploring the autistic experience and involving autistic people and people with learning disabilities in research.

\section{NOAH J. SASSON}

Noah Sasson is an Associate Professor of Psychology at the University of Texas at Dallas. He teaches college students, trains future scientists, and leads a research laboratory that tries to better understand why autistic people often experience social difficulties. 